\title{
An expected polynomial time algorithm for coloring 2-colorable 3-graphs $\|^{\dagger}$
}

\author{
Yury Person 俳 \\ Mathias Schacht蚛 \\ ${ }^{1}$ Institut für Mathematik, Freie Universität Berlin, Germany \\ ${ }^{2}$ Fachbereich Mathematik, Universität Hamburg, Germany
}

We present an algorithm that for 2-colorable 3-uniform hypergraphs, finds a 2-coloring in average running time $O\left(n^{5} \log ^{2} n\right)$.

Keywords: coloring, hypergraphs, average case analysis

\section{Introduction}

One of the classical problems in complexity theory is to decide whether a given $k$-uniform hypergraph is 2-colorable (or bipartite). Here a hypergraph is said to be $\ell$-colorable, if there is a coloring of its vertices with $\ell$ colors, such that no hyperedge is monochromatic. We call such coloring proper. The chromatic number of the hypergraph is defined to be the minimal number of colors needed to color a hypergraph properly.

While for bipartite graphs a 2-coloring can be found in linear time, it was shown by Lovász [16] that the problem becomes $N P$-complete for $k$-uniform hypergraphs and $k \geq 3$. Moreover, Guruswami et al. [9] proved that it is $N P$-hard to color bipartite, $k$-uniform hypergraphs with a constant number of colors for $k \geq 4$. It was also shown by Dinur et al. [3] that this problem remains inapproximable by a constant for 3-uniform hypergraphs. On the other hand, recently, Krivelevich et al. [15] found a polynomial time algorithm which colors 3-uniform bipartite hypergraphs using $O\left(n^{1 / 5} \log ^{c} n\right)$ colors. Another positive result is due to Chen and Frieze [1]. Those authors studied colorings of so-called $\alpha$-dense bipartite 3 uniform hypergraphs, where a 3 -uniform hypergraph is $\alpha$-dense if the joint degree of any two vertices is at least $\alpha n$. A randomized algorithm that can color $H$ in $n^{O(1 / \alpha)}$ time was found [1].

The purpose of this paper is to present an algorithm that colors a hypergraph chosen uniformly at random from the family of all labelled, 3-uniform, bipartite hypergraphs on $n$ vertices in $O\left(n^{5} \log ^{2} n\right)$ expected time. Indeed, we prove a slightly more general result for the class of Fano-free hypergraphs, see Theorem 1 Before we state it precisely we review related results for graphs.

\footnotetext{
${ }^{\dagger}$ A preliminary version of this article appeared in the Proceedings of Eurocomb 2009 [18].

‡Email: person@math. fu-berlin. de. Supported by GIF grant no. I-889-182.6/2005.

§Email: schacht@math.uni-hamburg.de
} 
In 1984 Wilf [22] noted, using a simple counting argument, that one can decide in constant expected time, whether a graph is $\ell$-colorable. Few years later Turner [21] gave an $O(|V|+|E| \log \ell)$ algorithm for optimally coloring almost all $\ell$-colorable graphs. This result was further expanded by Dyer and Frieze [4] who developed an algorithm which colored every $\ell$-colorable graph on $n$ vertices properly (with $\ell$ colors) in $O\left(n^{2}\right)$ expected time.

Another line of research concerns the study of monotone properties of the type $\operatorname{Forb}(n, L)$ for a fixed graph $L$, i.e., the family of all labeled graphs on $n$ vertices, which contain no copy of $L$ as a (not necessarily induced) subgraph. Prömel and Steger [19] gave an algorithm that colors properly (regardless of the value of its chromatic number $\chi(G))$ a randomly chosen member $G$ from $\operatorname{Forb}\left(n, K_{\ell+1}\right)$, i.e., the class of all labeled $K_{\ell+1}$-free graphs, in $O\left(n^{2}\right)$ expected time. This is clearly a generalization of the result of Dyer and Frieze in the light of the well known result of Kolaitis et al. [14] that almost all $K_{\ell+1}$-free graphs are $\ell$-colorable.

In [17] we studied $\operatorname{Forb}(n, F)$, where $F$ is the 3-uniform hypergraph of the Fano plane, which is the unique triple system with 7 hyperedges on 7 vertices where every pair of vertices is contained in precisely one hyperedge. It was shown independently by Füredi and Simonovits [8] and Keevash and Sudakov [11], that for large $n$ the unique extremal Fano-free hypergraph is the balanced, complete, bipartite hypergraph $B_{n}=\left(U \dot{\cup} W, E_{B_{n}}\right)$, where $|U|=\lfloor n / 2\rfloor,|W|=\lceil n / 2\rceil$ and $E_{B_{n}}$ consists of all hyperedges with at least one vertex in $U$ and one vertex in $W$. The hypergraph of the Fano plane $F$ is not bipartite, i.e., for every vertex partition $X \dot{\cup} Y=V(F)$ into two classes there exists an edge of $F$ which is either contained in $X$ or in $Y$. Consequently, $\operatorname{Forb}(n, F)$ contains any bipartite 3 -uniform hypergraph on $n$ vertices. However, deleting any hyperedge from $F$ results in a bipartite hypergraph.

Let $\mathcal{B}_{n}$ be the class of all labeled bipartite hypergraphs on $n$ vertices. It was shown in [17] that

$$
|\operatorname{Forb}(n, F)| \leq\left(1+2^{-\Omega\left(n^{2}\right)}\right)\left|\mathcal{B}_{n}\right|
$$

Our main result here states that one can color a 3-uniform hypergraph chosen uniformly at random from $\operatorname{Forb}(n, F)$ in polynomial expected time.

Theorem 1 There is an algorithm with average running time $O\left(n^{5} \log ^{2} n\right)$ which colors every member from $\operatorname{Forb}(n, F)$ properly.

Together with (1) we immediately derive in a similar manner to Steger and Prömel [19] that one can color a 3 -uniform hypergraph chosen uniformly at random from $\mathcal{B}_{n}$ in polynomial expected time.

Corollary 2 There is an algorithm with average running time $O\left(n^{5} \log ^{2} n\right)$ which finds a bipartition of every member from $\mathcal{B}_{n}$.

\section{Algorithmic version of the weak hypergraph regularity lemma}

In [17] we used the so-called weak hypergraph regularity lemma in conjuction with the recently discovered counting lemma for linear hypergraphs from [12]. A hypergraph is linear if any pair of hyperedges shares at most one vertex. Since the Fano plane is linear, this counting lemma is applicable.

Throughout this paper we will consider only 3 -uniform hypergraphs and by a hypergraph we will always mean a 3 -uniform hypergraph. For the sake of a simpler notation we set

$$
\mathcal{F}_{n}=\operatorname{Forb}(n, F) \text {, }
$$


where by $F$ we will always denote the hypergraph of the Fano plane. We will refer to hypergraphs not containing a copy of $F$, as Fano-free hypergraphs.

For a hypergraph $H=(V, E)$ and a set $U \subseteq V$ we denote by $H[U]$ the 3-uniform subhypergraph of $H$ induced on $U$. We write $E_{H}(U)$ or simply $E(U)$ for the edges completely contained in $U$, i.e., $E_{H}(U)=E \cap\left(\begin{array}{l}U \\ 3\end{array}\right)$. We let $e_{H}(U)$ (resp. $e(U)$ ) denote the cardinality of $E_{H}(U)$. Similarly, for two disjoint subsets $U$ and $W$ we write

$$
\begin{aligned}
E(U, W) & =E(U \cup W) \backslash(E(U) \cup E(W)) \\
& =\{e \in E: e \subset U \dot{\cup} W,|e \cap U||e \cap W| \geq 1\}
\end{aligned}
$$

and $e(U, W)=|E(U, W)|$. For pairwise disjoint sets $V_{1}, V_{2}$, and $V_{3}$ denote by $E_{H}\left(V_{1}, V_{2}, V_{3}\right)$ the set of all hyperedges from $H$ that intersect all three sets, further set $e_{H}\left(V_{1}, V_{2}, V_{3}\right)=\left|E_{H}\left(V_{1}, V_{2}, V_{3}\right)\right|$

The weak hypergraph regularity lemma is a straightforward extension of Szemerédi's regularity lemma [20] for graphs. Here we introduce it only for 3-uniform hypergraphs, but a similar lemma holds for general $k$. Let $H=(V, E)$ be a hypergraph and let $W_{1}, W_{2}, W_{3}$ be pairwise disjoint non-empty subsets of $V$. We denote by $d_{H}\left(W_{1}, W_{2}, W_{3}\right)=d\left(W_{1}, W_{2}, W_{3}\right)$ the density of the 3-partite induced subhypergraph $H\left[W_{1}, W_{2}, W_{3}\right]$ of $H$, defined by

$$
d_{H}\left(W_{1}, W_{2}, W_{3}\right)=\frac{e_{H}\left(W_{1}, W_{2}, W_{3}\right)}{\left|W_{1}\right|\left|W_{2}\right|\left|W_{3}\right|} .
$$

We say the triple $\left(V_{1}, V_{2}, V_{3}\right)$ of pairwise disjoint subsets $V_{1}, V_{2}, V_{3} \subseteq V$ is $(\varepsilon, d)$-regular, for $\varepsilon>0$ and $d \geq 0$, if

$$
\left|d_{H}\left(W_{1}, W_{2}, W_{3}\right)-d\right| \leq \varepsilon
$$

for all triples of subsets $W_{1} \subseteq V_{1}, W_{2} \subseteq V_{2}, W_{3} \subseteq V_{3}$ satisfying $\left|W_{i}\right| \geq \varepsilon\left|V_{i}\right|, i=1,2,3$. We say the triple $\left(V_{1}, V_{2}, V_{3}\right)$ is $\varepsilon$-regular if it is $(\varepsilon, d)$-regular for some $d \geq 0$. An $\varepsilon$-regular partition of a set $V(H)$ has the following properties:

(i) $V=V_{1} \dot{\cup} \ldots \dot{U} V_{t}$

(ii) ||$V_{i}|-| V_{j}|| \leq 1$ for all $1 \leq i, j \leq t$,

(iii) for all but at most $\varepsilon\left(\begin{array}{l}t \\ 3\end{array}\right)$ sets $\left\{i_{1}, i_{2}, i_{3}\right\} \subseteq[t]$, the triple $\left(V_{i_{1}}, V_{i_{2}}, V_{i_{3}}\right)$ is $\varepsilon$-regular.

We will apply an algorithmic version of the weak hypergraph regularity lemma which was established by Czygrinow and Rödl [2] and in a slightly adjusted form for our needs, it will be used here to analyze our coloring algorithm Color $(H)$, that will be introduced in the next section:

Theorem 3 (Algorithmic weak regularity lemma) For every integer $t_{0} \geq 1$ and every $\varepsilon>0$, there exist $T_{0}=T_{0}\left(t_{0}, \varepsilon\right), n_{0}=n_{0}\left(t_{0}, \varepsilon\right)$ and an algorithm Regularize, which for every hypergraph $H=(V, E)$ on $n \geq n_{0}$ vertices finds in $O\left(n^{5} \log ^{2} n\right)$ time an $\varepsilon$-regular partition $V=V_{1} \dot{\cup} \ldots \dot{\cup} V_{t}$ with $t_{0} \leq t \leq T_{0}$.

Typically, when studying the regular partition of a hypergraph, one defines a new hypergraph of bounded size with the vertex set being the partition classes and the edge set being $\varepsilon$-regular triples with sufficient density. Our algorithm will also work with such cluster-hypergraphs. The following definition makes this precise. 
Definition 4 For a hypergraph $H=(V, E)$ with an $\varepsilon$-regular partition $V(H)=V_{1} \dot{\cup} \ldots \dot{U} V_{t}$ of its vertex set and an $\eta>0$ let $H(\eta)=\left(V^{*}, E^{*}\right)$ be the cluster-hypergraph with vertex set $V^{*}=\{1, \ldots, t\}$ and edge set $E^{*}$, where for $1 \leq i<j<k \leq t$ it is $\{i, j, k\} \in E^{*}$ if and only if the triple $\left(V_{i}, V_{j}, V_{k}\right)$ is $\varepsilon$-regular and the density satisfies $d_{H}\left(V_{i}, V_{j}, V_{k}\right) \geq \eta$.

In [12] a counting lemma for linear hypergraphs in the context of the weak regularity lemma was proved. In [17] we noted that the same proof also holds for the lower bound and for a slightly relaxed definition of an $\varepsilon$-regular triple. More precisely, we say a triple $\left(V_{1}, V_{2}, V_{3}\right)$ of pairwise disjoint subsets $V_{1}, V_{2}, V_{3} \subseteq V$ is one-sided $(\varepsilon, d)$-regular for $\varepsilon>0$ and $d \geq 0$ if

$$
d_{H}\left(W_{1}, W_{2}, W_{3}\right) \geq d
$$

for all triples of subsets $W_{1} \subseteq V_{1}, W_{2} \subseteq V_{2}, W_{3} \subseteq V_{3}$ satisfying $\left|W_{i}\right| \geq \varepsilon\left|V_{i}\right|, i=1,2,3$. Note also, that an $(\varepsilon, d)$-regular triple is one-sided $(\varepsilon, d-\varepsilon)$-regular.

Theorem 5 (Key-Lemma (Theorem 3.3 in [17])) For every $\ell \in \mathbb{N}$ and $d>0$ there exist $\varepsilon=\varepsilon(\ell, d)>0$ and a positive integer $m_{0}=m_{0}(\ell, d)$ with the following property.

If $H$ is an $\ell$-partite 3 -uniform hypergraph with vertex classes $V_{1}, \ldots, V_{\ell}$, such that $\left|V_{1}\right|=\ldots=\left|V_{\ell}\right| \geq$ $m_{0}$, and $L$ is a linear hypergraph on $\ell$ vertices such that for every $e \in E(L)$ the triple $\left(V_{i}\right)_{i \in e}$ is one-sided $(\varepsilon, d)$-regular. Then $H$ contains a copy of $L$.

The proof of Theorem 5 is given in [17, Theorem 3.3] and, in fact, it follows along the lines of the proof of the counting lemma for linear hypergraphs in [12].

\section{Algorithm for coloring Fano-free hypergraphs}

Below we first present the simple algorithm $\operatorname{Color}(H)$ which is based on the subroutine $\operatorname{Partition}(H, \alpha)$.

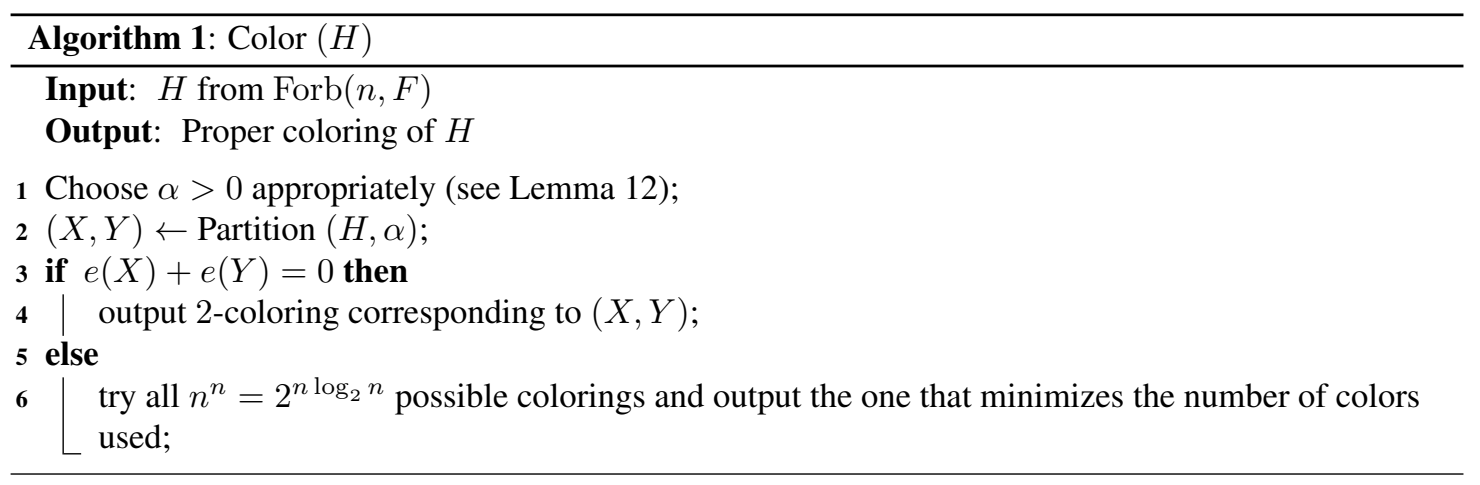

Obviously, $\operatorname{Color}(H)$ finds a proper coloring of $H$. We will show that Step 2 has a running time of $O\left(n^{5} \log ^{2} n\right)$ for all $H$. Hence for proving Theorem 1 it suffices to show that there exists an $\alpha>0$ such that Step 5 of the algorithm will be executed for at most $2^{-n \log _{2} n}|\operatorname{Forb}(n, F)| 3$-uniform hypergraphs from $\operatorname{Forb}(n, F)$. 
The subroutine Partition $(H, \alpha)$ finds a locally minimal partition $X_{H} \dot{\cup} Y_{H}=V(H)$, i.e., a partition for which $e\left(X_{H}\right)+e\left(Y_{H}\right)$ cannot be decreased by moving a single vertex from one class to another. Moreover, we will show later that for "most" 3-uniform hypergraphs $H$ from $\operatorname{Forb}(n, F)$ the algorithm Partition $(H, \alpha)$ outputs a partition with the additional property $e\left(X_{H}\right)+e\left(Y_{H}\right)<\alpha n^{3}$.

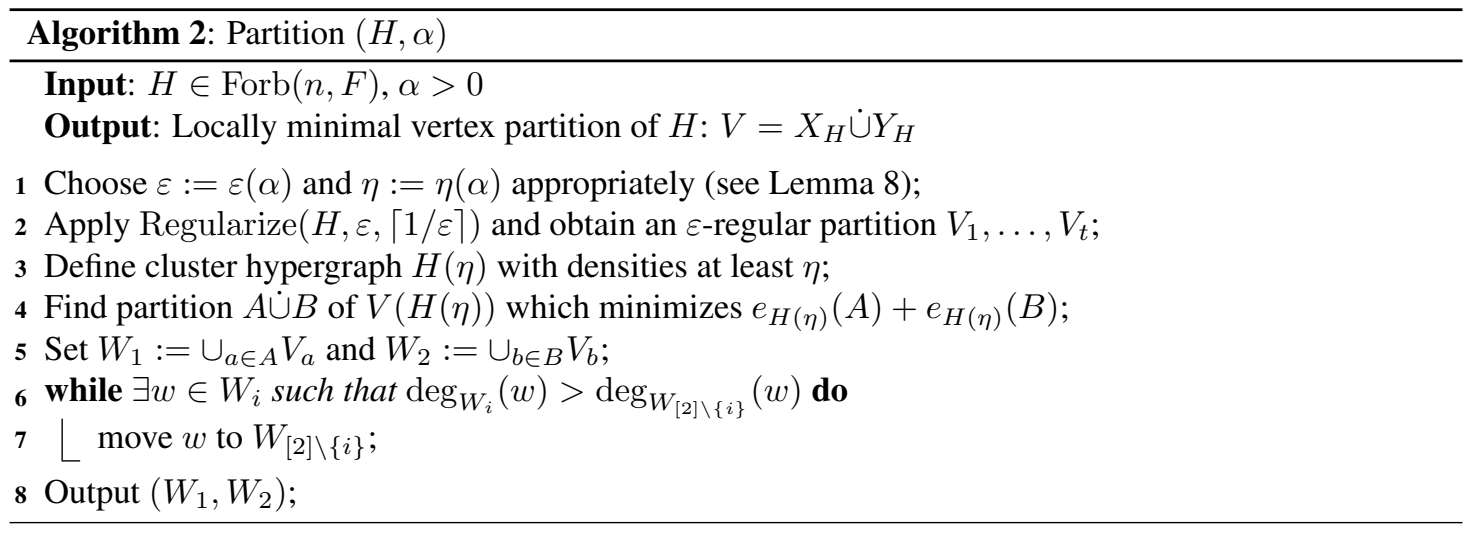

In Step 2 the algorithm Regularize $(H, \varepsilon, t)$ will be used as a subroutine. This algorithm, due to Czygrinow and Rödl [2], finds an $\varepsilon$-regular partition of a 3-uniform hypergraph $H$ on $n$ vertices and at least $t_{0}$ many clusters in time $O\left(n^{5} \log ^{2} n\right)$. Step 4 requires only constant time, that depends on $\varepsilon$ only. The "while"-loop also requires at most $O\left(n^{5}\right)$ steps, as the update step requires at most $O\left(n^{2}\right)$ operations and the "while"-loop terminates after at most $\left(\begin{array}{l}n \\ 3\end{array}\right)$ executions. Indeed, in every loop we increase the cut and there are at most $\left(\begin{array}{l}n \\ 3\end{array}\right)$ hyperedges in $H$.

\section{Further tools}

In the following we will introduce more notations and basic facts before we start with the analysis of the algorithm Color $(H)$. We recall the result of Füredi and Simonovits [8] and Keevash and Sudakov [11] on the extremal number for the hypergraph of the Fano plane $F$ asserts for sufficiently large $n$ :

$$
\operatorname{ex}(n, F)=e\left(B_{n}\right)=\left|E_{B_{n}}\right|=\left(\begin{array}{l}
n \\
3
\end{array}\right)-\left(\begin{array}{c}
\lceil n / 2\rceil \\
3
\end{array}\right)-\left(\begin{array}{c}
\lfloor n / 2\rfloor \\
3
\end{array}\right)=\frac{n^{3}}{8}-\frac{n^{2}}{4}-O(n) \leq \frac{n^{3}}{8} .
$$

The following simple bounds on $\left|\mathcal{B}_{n}\right|$ will be used:

$$
2^{e\left(B_{n}\right)} \leq\left|\mathcal{B}_{n}\right| \leq 2^{n} \cdot 2^{e\left(B_{n}\right)} .
$$

Indeed there are at most $2^{n}$ partitions of $[n]$ in two disjoint sets and there are at most $e\left(B_{n}\right)$ hyperedges running between those two sets.

The following holds for the Fano-extremal hypergraph $B_{n}$ :

$$
\delta_{1}\left(B_{n}\right)=\left(\left\lceil\frac{n}{2}\right\rceil-1\right)\left\lfloor\frac{n}{2}\right\rfloor+\left(\begin{array}{c}
\lfloor n / 2\rfloor \\
2
\end{array}\right) \geq \frac{3}{8} n^{2}-n,
$$


where for a hypergraph $H=(V, E)$ we denote by $\delta_{1}(H)$ the minimum vertex degree, i.e.,

$$
\delta_{1}(H)=\min _{u \in V}|\{\{v, w\}:\{u, v, w\} \in E\}|
$$

while setting

$$
\Delta(H)=\max _{u \in V}|\{\{v, w\}:\{u, v, w\} \in E\}|
$$

to be the maximum degree of $H$. Furthermore, we have

$$
e\left(B_{n}\right)=e\left(B_{n-3}\right)+\delta_{1}\left(B_{n}\right)+\delta_{1}\left(B_{n-1}\right)+\delta_{1}\left(B_{n-2}\right) .
$$

For a given hypergraph $H=(V, E)$ and a vertex $v \in V$ we define its link $L_{H}(v)=\left(V \backslash\{v\}, E_{v}\right)$ to be the graph whose edges together with $v$ form hyperedges of $H$, namely

$$
E_{v}=\{\{u, w\}:\{v, u, w\} \in E\} .
$$

We define the degree of $v \in V$ to be $\operatorname{deg}(v)=\operatorname{deg}_{H}(v)=\left|E\left(L_{H}(v)\right)\right|$, and sometimes we omit $H$ when it is clear from the context. For $A \subseteq V(H)$ and $v \in V(H)$ denote by

$$
L_{A}(v)=L_{H}(v)[A]=\left(A \backslash\{v\}, E_{v} \cap\left(\begin{array}{c}
A \\
2
\end{array}\right)\right)
$$

the link of $v$ induced on $A$.

The following stability result for Fano-free hypergraphs was proved by Keevash and Sudakov [11] and Füredi and Simonovits [8], in order to determine the extremal hypergraph for the Fano plane:

Theorem 6 (Stability theorem for Fano-free hypergraphs) For every $\alpha>0$ there exists $\lambda>0$ and $n_{0}$ such that for every Fano-free hypergraph $H$ on $n \geq n_{0}$ vertices with at least $\left(\frac{1}{8}-\lambda\right) n^{3}$ hyperedges there exists a partition $V(H)=X \dot{\cup} Y$ so that $e(X)+e(Y)<\alpha n^{3}$.

It follows from the work in [8, 11] that $\lambda=\lambda(\alpha)$ is indeed a computable function.

By $h(x)=-x \log x-(1-x) \log (1-x)$ we denote the entropy function, and by $\log$ we always mean $\log _{2}$. Note further, $h(x) \rightarrow 0$ as $x$ tends to 0 , and $h(x) \geq x$ for $x \leq \frac{1}{2}$. We will use the entropy function $h(x)$ together with the well-known inequality

$$
\left(\begin{array}{c}
n \\
x n
\end{array}\right) \leq 2^{h(x) n}
$$

which holds for $0<x<1$. Furthermore, we will use that for $n>3 k$ we have $\sum_{j<k}\left(\begin{array}{l}n \\ j\end{array}\right)<\left(\begin{array}{l}n \\ k\end{array}\right)$. Often we will omit floors and ceilings, as they will have no effect on our asymptotic arguments. 


\section{Proof of main result}

\subsection{Overview of the proof}

So far we have presented our coloring algorithm $\operatorname{Color}(H)$ and it is left to show that there exist appropriate choices for $\alpha$ and for $\varepsilon$ and $\eta$ inside the subroutine $\operatorname{Partition}(H, \alpha)$ which yield the claimed running time. More precisely, we will show that for sufficiently small $\alpha, \varepsilon$ and $\eta$ the proportion of hypergraphs $H$ in $\operatorname{Forb}(n, F)$ for which Step 6 in Color $(H)$ is required, is "negligible".

In the main part of the proof we show that there are at most $2^{-\Omega\left(n^{2}\right)}|\operatorname{Forb}(n, F)|$ such hypergraphs in $\operatorname{Forb}(n, F)$. To prove this, we study structural properties of a typical $H$ from $\operatorname{Forb}(n, F)$. Our analysis uses ideas from [17]. We will introduce a chain of subsets of $\operatorname{Forb}(n, F)$ such that all members of them possess certain "typical" properties. More precisely, we study the following chains of subsets of $\mathcal{F}_{n}$ :

$$
\mathcal{F}_{n} \supseteq \mathcal{F}_{n}^{\prime}(\alpha) \supseteq \mathcal{F}_{n}^{\prime \prime}(\alpha, \beta) \supseteq \mathcal{F}_{n}^{\prime \prime \prime}(\alpha, \beta),
$$

and

$$
\mathcal{F}_{n} \supseteq \mathcal{B}_{n} \supseteq \mathcal{F}_{n}^{\prime \prime \prime}(\alpha, \beta) .
$$

The first subset $\mathcal{F}_{n}^{\prime}(\alpha)$ consists of those members, that admit at least one locally minimal partition $\left(X_{H}, Y_{H}\right)$ with the properties $e\left(X_{H}\right)+e\left(Y_{H}\right)<\alpha n^{3}$ and $\left|X_{H}\right| \approx\left|Y_{H}\right|$. Using the weak hypergraph regularity lemma and Theorem 6, we will show that for most of the members from $\mathcal{F}_{n}^{\prime}(\alpha)$ the algorithm Partition $(H, \alpha)$ finds a locally minimal partition for given $\alpha$. Therefore, additionally, we obtain that most of the hypergraphs from $\operatorname{Forb}(n, F)$ lie in $\mathcal{F}_{n}^{\prime}(\alpha)$.

The further analysis proceeds as follows. We introduce two more proper subsets of $\operatorname{Forb}(n, F)$ : $\mathcal{F}_{n}^{\prime \prime}(\alpha, \beta)$ and $\mathcal{F}_{n}^{\prime \prime \prime}(\alpha, \beta)$ which describe two further "useful" properties of almost all Fano-free hypergraphs on $n$ vertices. The family $\mathcal{F}_{n}^{\prime \prime}(\alpha, \beta)$ contains those members from $\mathcal{F}_{n}^{\prime}(\alpha)$ which are "dense everywhere" in the sense that whenever we take three disjoint subsets of vertices, say $W_{1}, W_{2}, W_{3}$, not all of them contained in $X_{H}$ or $Y_{H}$ (for any locally minimal partition satisfying properties from $\mathcal{F}_{n}^{\prime}(\alpha)$ ), the number of hyperedges that run between them will be at least $d\left|W_{1}\right|\left|W_{2}\right|\left|W_{3}\right|$ for some positive constant $d>0$. Moreover, every vertex will have small degree in its own partition class (i.e. $X_{H}$ or $Y_{H}$ ). Thus, essentially, there exists "only one" locally minimal partition. For members of the last class $\mathcal{F}_{n}^{\prime \prime \prime}(\alpha, \beta)$ we demand that the joint link of every set of 3 vertices of any of the two partition classes $X_{H}$ and $Y_{H}$ must contain a $K_{4}$. We then deduce that the last property implies in fact bipartiteness. As a seemingly surprising fact, we obtain, that for almost all members from $\operatorname{Forb}(n, F)$ any locally minimal partition for some appropriate $\alpha$ already satisfies $e\left(X_{H}\right)+e\left(Y_{H}\right)=0$.

\subsection{Proof of Theorem 1}

Below we give proper definitions of the classes described above and we state the lemmas that relate the sizes of these hypergraph classes. The proofs of the corresponding statements are given in the next section, Section 6 First we recall the definition of a locally minimal partition. A vertex partition $X_{H} \dot{\cup} Y_{H}$ of $V(H)$ is locally minimal if $e\left(X^{\prime}, Y^{\prime}\right) \geq e\left(X_{H}, Y_{H}\right)$ for all partitions $X^{\prime} \dot{\cup} Y^{\prime}$ of $V(H)$ with $\left|X^{\prime} \Delta X_{H}\right| \leq 1$. Furthermore, we say a partition is $\alpha$-good, if $e\left(X_{H}\right)+e\left(Y_{H}\right)<\alpha n^{3}$ and $\left|X_{H}\right|,\left|Y_{H}\right|<n / 2+2 \sqrt{h(6 \alpha)} n$. The first class $\mathcal{F}_{n}^{\prime}(\alpha)$ of Fano-free hypergraphs is defined as follows.

Definition 7 Let $\alpha>0$ and $n \in \mathbb{N}$. We set

$$
\mathcal{F}_{n}^{\prime}(\alpha)=\left\{H \in \mathcal{F}_{n}: \exists \text { a locally minimal } \alpha \text {-good partition } V=X_{H} \dot{\cup} Y_{H}\right\} .
$$


Lemma 8 For every $\alpha \in\left(0, \frac{1}{12}\right)$ there exist (computable) constants $c^{\prime}, \varepsilon, \eta>0$ and an integer $n_{0}^{\prime}$ such that for all $n \geq n_{0}^{\prime}$ the algorithm $\operatorname{Partition}(H, \alpha)$ finds for all but at most $2^{e\left(B_{n}\right)-c^{\prime} n^{3}}$ hypergraphs $H \in \mathcal{F}_{n}$ a locally minimal partition $X_{H} \cup \dot{\cup} Y_{H}$ of its vertex set with the following two properties:

- $e\left(X_{H}\right)+e\left(Y_{H}\right)<\alpha n^{3}$,

- $\left|X_{H}\right|,\left|Y_{H}\right|<n / 2+2 \sqrt{h(6 \alpha)} n$.

In particular, we have:

$$
\left|\mathcal{F}_{n} \backslash \mathcal{F}_{n}^{\prime}(\alpha)\right|<2^{e\left(B_{n}\right)-c^{\prime} n^{3}} .
$$

Next we define the subfamily of "everywhere dense" hypergraphs from $\mathcal{F}_{n}^{\prime}(\alpha)$.

Definition 9 For $\alpha, \beta>0$ and $n \in \mathbb{N}$ let $\mathcal{F}_{n}^{\prime \prime}(\alpha, \beta)$ denote the family of those hypergraphs $H \in \mathcal{F}_{n}^{\prime}(\alpha)$, such that for any locally minimal $\alpha$-good partition $X_{H} \cup Y_{H}$ of $V(H)$ the following condition holds.

For any pairwise disjoint sets $W_{1} \subset X_{H}, W_{2} \subset Y_{H}$ and $W_{3} \subset Z_{H}$, where $Z_{H} \in\left\{X_{H}, Y_{H}\right\}$, with $\left|W_{i}\right| \geq \beta$ for $i=1,2,3$ we have

$$
e_{H}\left(W_{1}, W_{2}, W_{3}\right) \geq \frac{1}{4}\left|W_{1}\right|\left|W_{2}\right|\left|W_{3}\right| .
$$

The proof of the following lemma is similar to the proofs of Lemmas 4.2 and 4.3 in [17], for completeness we include the proof of Lemma 10 in Section 6.

Lemma 10 For every $\gamma>0$ there exist (computable) constants $\alpha, \beta, c^{\prime \prime}>0$ and an integer $n_{0}$ such that for every $n \geq n_{0}$ and $H \in \mathcal{F}_{n}^{\prime \prime}(\alpha, \beta)$, we have:

1. $\left|\mathcal{F}_{n}^{\prime}(\alpha) \backslash \mathcal{F}_{n}^{\prime \prime}(\alpha, \beta)\right|<2^{e\left(B_{n}\right)-c^{\prime \prime} n^{3}}$.

2. $\max \left\{\Delta\left(H\left[X_{H}\right]\right), \Delta\left(H\left[Y_{H}\right]\right)\right\}<\gamma n^{2}$ for any locally minimal $\alpha$-good partition $X_{H} \dot{\cup} Y_{H}$,

We finally define the last subclass of Fano-free hypergraphs.

Definition 11 For $\alpha, \beta>0$ and $n \in \mathbb{N}$, let $\mathcal{F}_{n}^{\prime \prime \prime}(\alpha, \beta)$ denote the family of those hypergraphs $H \in$ $\mathcal{F}_{n}^{\prime \prime}(\alpha, \beta)$, such that for any locally minimal $\alpha$-good partition $X_{H} \cup Y_{H}$ of $V(H)$ the following holds.

For all triples $z_{1}, z_{2}, z_{3} \in Z$ of vertices with $Z \in\left\{X_{H}, Y_{H}\right\}$ we have $L_{Q}\left(z_{1}\right) \cap L_{Q}\left(z_{2}\right) \cap L_{Q}\left(z_{3}\right) \supseteq K_{4}$, where $\{Q, Z\}=\left\{X_{H}, Y_{H}\right\}$. In other words, we require that the common link of any triple from $X_{H}$ or $Y_{H}$ contains a copy of $K_{4}$ in the other vertex class.

It follows directly from the definition, that every $H \in \mathcal{F}_{n}^{\prime \prime \prime}(\alpha, \beta)$ is bipartite, i.e., $\mathcal{F}_{n}^{\prime \prime \prime}(\alpha, \beta) \subseteq \mathcal{B}_{n}$. Otherwise, any hyperedge $e$, say in $X_{H}$, together with the $K_{4}$ in $Y_{H}$, which lies in the common link of the vertices of $e$ would span a copy of the hypergraph of the Fano plane.

To obtain a bound on $\left|\mathcal{F}_{n}^{\prime \prime}(\alpha, \beta) \backslash \mathcal{F}_{n}^{\prime \prime \prime}(\alpha, \beta)\right|$ we estimate in at most how many ways one can construct a Fano-free hypergraph from $\mathcal{F}_{n}^{\prime \prime}(\alpha, \beta) \backslash \mathcal{F}_{n}^{\prime \prime \prime}(\alpha, \beta)$, i.e. a Fano-free hypergraph with a locally minimal partition and a hyperedge inside it.

Lemma 12 There exist (computable) constants $\alpha, \beta, c>0$ and an integer $n_{0}$, such that for every $n \geq n_{0}$ we have

$$
\left|\mathcal{F}_{n}^{\prime}(\alpha) \backslash \mathcal{F}_{n}^{\prime \prime \prime}(\alpha, \beta)\right| \leq 2^{e\left(B_{n}\right)-c n^{2}} .
$$


We remark that the bound in Lemma 12 is considerably weaker than those in Lemma 10 and Lemma 8 having only $-c n^{2}$ in the exponent instead of $-c n^{3}$. The reason for that is that the number of Fanofree hypergraphs which are not bipartite is $2^{e\left(B_{n}\right)-\Theta\left(n^{2}\right)}$. The corresponding argument can be found in Section 7

Proof of Theorem 1; We first apply Lemma 12 and obtain constants $\alpha, \beta$ and $c$. Then Lemma 8 applied with $\alpha$ returns $\varepsilon, \eta$ and $c^{\prime}$. Below we show that these constants $\alpha, \varepsilon$ and $\eta$ are suitable choices in the algorithms Color and Partition.

Indeed for these choices of $\alpha, \varepsilon$ and $\eta$, Lemma 8 asserts that the partition $X_{H} \cup Y_{H}$ provided by Partition $(H, \alpha)$ is locally minimal and $\alpha$-good for all but $2^{e\left(B_{n}\right)-c^{\prime} n^{3}}$ hypergraphs $H \in \mathcal{F}_{n}$. Moreover, due to Lemma 12 this partition satisfies $e\left(X_{H}\right)+e\left(Y_{H}\right)=0$ for all $H \in \mathcal{F}_{n}^{\prime \prime \prime}(\alpha, \beta)$, i.e. this partition is a correct 2-coloring of $H$. Finally, it follows from Lemma 8 and Lemma 12 that Step 6 of the algorithm Color is only considered for at most

$$
2^{e\left(B_{n}\right)-c^{\prime} n^{3}}+2^{e\left(B_{n}\right)-c n^{2}}
$$

Fano-free hypergraphs.

\section{Proofs of Lemmas}

Proof of Lemma 8; The proof of Lemma 8 combines the weak hypergraph regularity lemma with the stability theorem for Fano-free hypergraphs applied to the cluster-hypergraph.

Let $\lambda=\lambda(\alpha / 2)$ and $n_{0}(\alpha / 2)$ be given by the stability theorem, Theorem 6 We may assume $\lambda<$ $16 h(6 \alpha)$. We set

$$
c^{\prime}=\frac{\lambda}{17}
$$

and we choose $\eta$ such that $\lambda>(16 / 3) h(6 \eta)$ and $\eta \leq \alpha / 2$.

Later we will apply the Key-lemma, Theorem 5, with $\ell=7$ and $L$ being the hypergraph of the Fano plane to the cluster-hypergraph of an $\varepsilon$-regular partition. For that we set $\varepsilon=\min \{\varepsilon(7, \eta / 2), \eta / 2\}$, where $\varepsilon(7, \eta / 2)$ is as asserted by Theorem 5 .

We also choose a lower bound on the number of clusters in an $\varepsilon$-regular partition to be

$$
t_{0}=\max \left\{1 / \varepsilon, n_{0}(\alpha / 2)\right\},
$$

where $n_{0}(\alpha / 2)$ is the bound given by Theorem 6 , so that we can later apply Theorem 6 (stability theorem) to the cluster-hypergraph. Finally set $n$ be sufficiently large, in particular, set $n_{0}^{\prime} \gg \max \left\{T_{0}, n_{0}\right\}$, where $T_{0}$ and $n_{0}$ are given by the weak regularity lemma, Theorem 3 , which in turn will deliver an $\varepsilon$-regular partition later.

For the main steps of the proof it is sufficient to keep in mind that

$$
0<1 / t_{0} \leq \varepsilon \leq \eta \ll \lambda \ll \alpha .
$$

We may assume in the following that $t$ divides $n$, and thus $\left|V_{i}\right|=n / t$ for all $i=1, \ldots, t$.

We will upper bound the number of hypergraphs for which $\operatorname{Partition}(H, \alpha)$ fails to produce a locally minimal $\alpha$-good partition in two steps. More formally, we consider the subset $\tilde{\mathcal{F}}_{n}^{\prime}(\alpha)$ which consists of 
those hypergraphs $H$ from $\mathcal{F}_{n}$, for which $\operatorname{Partition}(H, \alpha)$ returns a locally minimal $\alpha$-good partition. Thus, our aim is to show that $\left|\mathcal{F}_{n} \backslash \tilde{\mathcal{F}}_{n}^{\prime}(\alpha)\right|$ is at most $2^{e\left(B_{n}\right)-c^{\prime} n^{3}}$. Our proof has two steps.

In the first step we bound the number of hypergraphs $H$ that have $e\left(X_{H}\right)+e\left(Y_{H}\right) \geq \alpha n^{3}$ for every locally minimal partition $\left(X_{H}, Y_{H}\right)$. In the second step we show that most of the hypergraphs $H$ every locally minimal partition $X_{H} \dot{\cup} Y_{H}$ with $e\left(X_{H}\right)+e\left(Y_{H}\right)<\alpha n^{3}$ will also satisfy:

$$
\max \left\{\left|X_{H}\right|,\left|Y_{H}\right|\right\}<\frac{n}{2}+2 \sqrt{h(6 \alpha)} n .
$$

Here and in the following $\left(X_{H}, Y_{H}\right)$ will stand for a locally minimal partition, and unless it is specified otherwise, it will stand for an arbitrary locally minimal partition.

Step 1. Consider a hypergraph $H \in \mathcal{F}_{n}$ satisfying

$$
e\left(X_{H}\right)+e\left(Y_{H}\right) \geq \alpha n^{3}
$$

for every locally minimal partition $X_{H} \cup \dot{\cup} Y_{H}$. We apply the weak regularity lemma, Theorem 3 , with parameters $\varepsilon$ and $t_{0}$. Firstly, we estimate the number of hyperedges, which are contained in the "uncontrolled" part of the regular partition:

- the number of hyperedges intersecting at most two of the clusters is at most

$$
t\left(\begin{array}{c}
n / t \\
2
\end{array}\right) n<\frac{1}{2 t} n^{3}
$$

- the number of hyperedges contained in irregular triples is at most

$$
\varepsilon\left(\begin{array}{l}
t \\
3
\end{array}\right)\left(\frac{n}{t}\right)^{3}<\frac{\varepsilon}{6} n^{3}
$$

- the number of hyperedges that are contained in $\varepsilon$-regular triples of density less than $\eta$ is at most

$$
\eta\left(\frac{n}{t}\right)^{3}\left(\begin{array}{l}
t \\
3
\end{array}\right)<\frac{\eta}{6} n^{3}
$$

Thus, the number of discarded edges is less than $\eta n^{3}$.

Secondly, consider the resulting cluster-hypergraph $H(\eta)$. It must be Fano-free as otherwise Theorem 5 would imply that $H$ also contains a copy of the hypergraph of the Fano plane.

Recall that by assumption (4) we have for every locally minimal partition $e\left(X_{H}\right)+e\left(Y_{H}\right) \geq \alpha n^{3}$. Below we will show that this assumption implies that $e(H(\eta)) \leq(1-\lambda) t^{3} / 8$. In fact, assuming the contrary, Theorem 6 applied to $H(\eta)$ would yield the existence of a bipartition of $\{1, \ldots, t\}$ into disjoint sets $A$ and $B$ with $e_{H(\eta)}(A)+e_{H(\eta)}(B)<\alpha t^{3} / 2$. We define the following bipartition of $V(H)$

$$
X=\bigcup_{i \in A} V_{i} \quad \text { and } \quad Y=\bigcup_{j \in B} V_{j} .
$$

Clearly, we then have

$$
e_{H}(X)+e_{H}(Y)<\eta n^{3}+\frac{\alpha}{2} t^{3}\left(\frac{n}{t}\right)^{3}<\alpha n^{3}
$$


Note that if shifting vertices between $X$ and $Y$ until a locally minimal partition is reached, only decreases the number of edges within classes. Thus, we would arrive at a locally minimal bipartition $\left(X^{\prime}, Y^{\prime}\right)$ with $e_{H}\left(X^{\prime}\right)+e_{H}\left(Y^{\prime}\right)<\alpha n^{3}$, which yields a contradiction to (4).

Now we are able to bound the number of hypergraphs $H \in \mathcal{F}_{n}$ with $e\left(X_{H}\right)+e\left(Y_{H}\right) \geq \alpha n^{3}$ for every locally minimal partition $X_{H} \dot{\cup} Y_{H}$ from above by calculating the total possible number of $\varepsilon$-regular partitions together with all possible cluster-hypergraphs associated with them and all possible hypergraphs that could give rise to such a particular cluster-hypergraph. This way we get, for sufficiently large $n$, due to the choice of $\eta$, at most

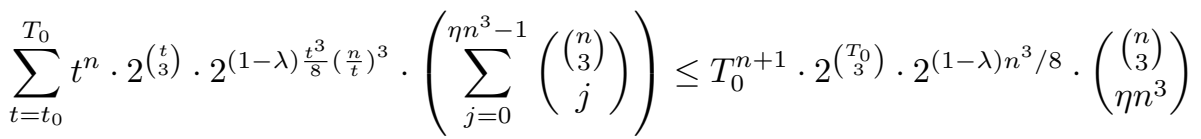

$$
\begin{aligned}
& \leq 2^{(n+1) \log T_{0}+\left(\begin{array}{c}
T_{0} \\
3
\end{array}\right)+n^{3} / 8-\lambda n^{3} / 8+h(6 \eta) n^{3} / 6}<2^{n^{3} / 8-\lambda n^{3} / 16},
\end{aligned}
$$

hypergraphs whose every locally minimal partition $\left(X_{H}, Y_{H}\right)$ satisfies (4).

Step 2. We now estimate the number of those hypergraphs $H$ which have a locally minimal partition $\left(X_{H}, Y_{H}\right)$ with $e\left(X_{H}\right)+e\left(Y_{H}\right)<\alpha n^{3}$, but

$$
\max \left\{\left|X_{H}\right|,\left|Y_{H}\right|\right\} \geq n / 2+2 \sqrt{h(6 \alpha)} n .
$$

First we upper bound $e\left(X_{H}, Y_{H}\right)$ for such a hypergraph $H$ by

$$
\begin{aligned}
e\left(X_{H}, Y_{H}\right) & \leq\left|X_{H}\right|\left(\begin{array}{c}
\left|Y_{H}\right| \\
2
\end{array}\right)+\left|Y_{H}\right|\left(\begin{array}{c}
\left|X_{H}\right| \\
2
\end{array}\right) \\
& <\frac{n}{2}\left|X_{H}\right|\left|Y_{H}\right|<\frac{n^{3}}{8}-2 h(6 \alpha) n^{3} .
\end{aligned}
$$

Note that there are at most $2^{n}$ possible partitions of $V(H)$, and since less than $\alpha n^{3}$ hyperedges are completely contained in $X_{H}$ and $Y_{H}$, those hyperedges can be chosen in at most

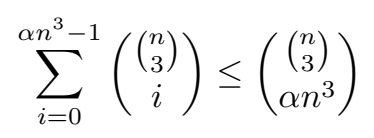

ways. Finally, as we assumed that the partitions satisfy (5), we estimate the number of possible choices of hyperedges between $X_{H}$ and $Y_{H}$ by $2^{n^{3} / 8-2 h(6 \alpha) n^{3}}$. Altogether we get, that there are at most

$$
2^{n} \cdot\left(\begin{array}{c}
\left(\begin{array}{c}
n \\
3
\end{array}\right) \\
\alpha n^{3}
\end{array}\right) \cdot 2^{n^{3} / 8-2 h(6 \alpha) n^{3}} \leq 2^{n+h(6 \alpha) n^{3} / 6+n^{3} / 8-2 h(6 \alpha) n^{3}} \leq 2^{n^{3} / 8-h(6 \alpha) n^{3}}
$$

hypergraphs $H$ with $e\left(X_{H}\right)+e\left(Y_{H}\right)<\alpha n^{3}$ and

$$
\max \left\{\left|X_{H}\right|,\left|Y_{H}\right|\right\} \geq \frac{n}{2}+2 \sqrt{h(6 \alpha)} n .
$$

Combining Steps 1 and 2 we obtain:

$$
\left|\mathcal{F}_{n} \backslash \mathcal{F}_{n}^{\prime}(\alpha)\right| \leq\left|\mathcal{F}_{n} \backslash \tilde{\mathcal{F}}_{n}^{\prime}(\alpha)\right| \leq 2^{n^{3} / 8-\lambda n^{3} / 16}+2^{n^{3} / 8-h(6 \alpha) n^{3}}<2^{n^{3} / 8-\lambda n^{3} / 16+1}
$$


since $h(6 \alpha)>\lambda / 16$. Due to $n^{3} / 8-e\left(B_{n}\right) \leq n^{2} / 4+O(n)$ and the choice of $c^{\prime}=\lambda / 17$, the lemma follows for sufficiently large $n$.

To prove Lemma 10 we will use a special form of the regularity lemma for graphs. Before we state it, we briefly introduce the concept of $\varepsilon$-regularity for graphs.

For a bipartite graph $G=\left(V_{1}, V_{2}, E\right)$ we define the density of $G$ as $d\left(V_{1}, V_{2}\right):=\frac{|E|}{\left|V_{1}\right|\left|V_{2}\right|}$. The density of a subpair $\left(U_{1}, U_{2}\right)$, where $U_{i} \subseteq V_{i}$ for $i=1,2$ is defined as $d\left(U_{1}, U_{2}\right):=\frac{e\left(U_{1}, U_{2}\right)}{\left|U_{1}\right|\left|U_{2}\right|}$. Here, as in the hypergraph case, $e\left(U_{1}, U_{2}\right)$ denotes the number of edges with one end in $U_{1}$ and the other in $U_{2}$. We say, $G$, or simply $\left(V_{1}, V_{2}\right)$, is $\varepsilon$-regular if for all $U_{i} \subseteq V_{i},\left|U_{i}\right| \geq\left|V_{i}\right|, i=1,2$ the following holds:

$$
\left|d\left(V_{1}, V_{2}\right)-d\left(U_{1}, U_{2}\right)\right|<\varepsilon .
$$

Now we can state the theorem, which is a straightforward consequence of the regularity lemma [20]:

Theorem 13 Let $\eta>0$, then for every $\varepsilon \in(0, \eta / 3)$ there exist $T_{0}, N_{0}$ such that the following holds. For all vertex disjoint graphs $G_{X}$ and $G_{Y}$ on $n \geq N_{0}$ vertices with $e\left(G_{X}\right), e\left(G_{Y}\right) \geq \eta n^{2}$ there exist $t \leq T_{0}$ and pairwise disjoint sets $X_{1}, X_{2}, Y_{1}, Y_{2}, Y_{3}, Y_{4}$, each of size $n / t$, and $X_{1}, X_{2} \subset V\left(G_{X}\right)$ and $Y_{i} \subset V\left(G_{Y}\right), i \in[4]$, so that $G_{X}\left[X_{1}, X_{2}\right], G_{Y}\left[Y_{1}, Y_{2}\right]$ and $G_{Y}\left[Y_{3}, Y_{4}\right]$ are $\varepsilon$-regular with density at least $\eta / 3$.

Proof of Lemma 10: For the second part of the proof, we let $\varepsilon=\min \left\{\frac{1}{2} \varepsilon(\gamma / 6), \gamma / 6\right\}$, where $\varepsilon(\gamma / 6)$ is as asserted by Theorem 5 . We choose $\beta=\varepsilon / T_{0}$, where $T_{0}$ is as asserted by Theorem 13 when applied with $\eta=\gamma$ and $\varepsilon$. Furthermore we choose $n_{0}$ to be sufficiently large, in particular larger than $N_{0}$.

For the first part, we choose $\alpha>0$ such that

$$
\beta^{3}\left(1-h\left(\frac{1}{4}\right)\right) \geq \frac{h(6 \alpha)}{3}
$$

and set $c^{\prime \prime}=h(6 \alpha) / 12$.

It is sufficient to keep in mind:

$$
0 \ll \alpha \ll \beta \ll \varepsilon \ll \gamma .
$$

(1) We estimate $\left|\mathcal{F}_{n}^{\prime}(\alpha) \backslash \mathcal{F}_{n}^{\prime \prime}(\alpha, \beta)\right|$ as follows. There are at most $2^{n}$ partitions $X_{H} \dot{\cup} Y_{H}=[n]$ of the vertex set and we can choose the edges lying completely within $X_{H}$ and $Y_{H}$ in at most

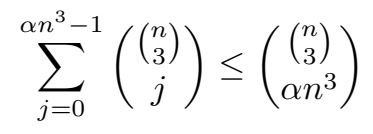

possible ways. Moreover, there are at most

$$
2\left(\begin{array}{c}
\frac{n}{2}+2 \sqrt{h(6 \alpha)} n \\
\beta n
\end{array}\right)^{3} \cdot \sum_{i=0}^{\frac{1}{4} \beta^{3} n^{3}-1}\left(\begin{array}{c}
\beta^{3} n^{3} \\
i
\end{array}\right)<2^{3 n+1}\left(\begin{array}{c}
\beta^{3} n^{3} \\
\frac{1}{4} \beta^{3} n^{3}
\end{array}\right)
$$

ways to select $W_{1}, W_{2}, W_{3}$ and the hyperedges in $e_{H}\left(W_{1}, W_{2}, W_{3}\right)$. Finally, there are at most

$$
2^{e\left(B_{n}\right)-\beta^{3} n^{3}}
$$


ways for choosing the remaining edges. Multiplying everything together, we obtain

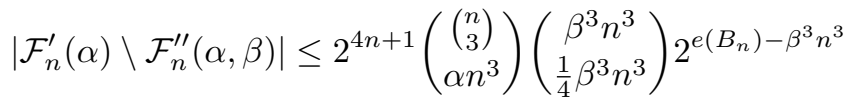

$$
\begin{aligned}
& \leq 2^{4 n+1+h(6 \alpha) \frac{n^{3}}{6}+h(1 / 4) \beta^{3} n^{3}+e\left(B_{n}\right)-\beta^{3} n^{3}} \\
& \leq 2^{e\left(B_{n}\right)-2 c^{\prime \prime} n^{3}}
\end{aligned}
$$

for sufficiently large $n$.

(2) We prove the second part by contradiction. More precisely, we will assume that there exists $H \in$ $\mathcal{F}_{n}^{\prime \prime}(\alpha, \beta)$ with $\max \left\{\Delta\left(H\left[X_{H}\right]\right), \Delta\left(H\left[Y_{H}\right]\right)\right\} \geq \gamma n^{2}$, and we will show that $H$ contains a Fano plane.

Without loss of generality we may assume that there exists $H \in \mathcal{F}_{n}^{\prime \prime}(\alpha, \beta)$ and a vertex $x \in X_{H}$ with $\operatorname{deg}_{H[X]}(x) \geq \gamma n^{2}$. Thus, $\left|L_{Y}(x)\right| \geq\left|L_{X}(x)\right| \geq \gamma n^{2}$, as otherwise this violates the minimality condition of the partition $X_{H} \dot{\cup} Y_{H}=V(H)$. Define graphs $G_{X}=\left(X_{H} \backslash\{x\}, L_{X}(x)\right)$ and $G_{Y}=\left(Y_{H}, L_{Y}(x)\right)$. We apply Theorem 13 to $G_{X} \cup \dot{\cup} G_{Y}$ and obtain $\varepsilon$-regular pairs $\left(X_{1}, X_{2}\right) \subset G_{X}$ and $\left(Y_{1}, Y_{2}\right),\left(Y_{3}, Y_{4}\right) \subset$ $G_{Y}$, with $\left|X_{i}\right|=\left|Y_{j}\right| \geq(n-1) / T_{0}$ and $i \in[2], j \in[4]$, each of density at least $\gamma / 3$.

Consider the following 7-partite subhypergraph $L$ with vertex classes $\{x\}, X_{1}, X_{2}, Y_{1}, Y_{2}, Y_{3}$ and $Y_{4}$. Denote $L^{x}$ to be the hypergraph obtained from $L$ by blowing up its first vertex class $\{x\}$ to the size of $X_{1}$ (all other partition classes are equal), and denote this blown-up class by $\tilde{X}$. More precisely, $L^{x}=\left(W^{x}, E^{x}\right)$, where

$$
W^{x}=\tilde{X} \dot{\cup} X_{1} \dot{\cup} X_{2} \dot{\cup} Y_{1} \dot{\cup} Y_{2} \dot{\cup} Y_{3} \dot{\cup} Y_{4}
$$

and

$$
\{a, b, c\} \in E^{x} \Leftrightarrow \begin{cases}\{a, b, c\} \in E(L), & \text { if }\{a, b, c\} \cap \tilde{X}=\emptyset, \\ \{x, b, c\} \in E(L), & \text { if } a \in \tilde{X} \text { and } b, c \notin \tilde{X} .\end{cases}
$$

Note, that $L$ contains a Fano plane if, and only if $L^{x}$ contains one. Now we apply Theorem 5 to $L^{x}$, as $L^{x}$ contains now 7 one-sided $(\varepsilon, \gamma / 6)$-regular triples and these triples form a Fano plane. This is true since the triples $\left(\tilde{X}, X_{1}, X_{2}\right),\left(\tilde{X}, Y_{1}, Y_{2}\right)$ and $\left(\tilde{X}, Y_{3}, Y_{4}\right)$ inherit the $\varepsilon$-regularity from the $\varepsilon$-regular pairs of $\left(X_{1}, X_{2}\right),\left(Y_{1}, Y_{2}\right)$, and $\left(Y_{3}, Y_{4}\right)$, while the other triples are one-sided $(\varepsilon, \gamma / 6)$-regular due to the choice of $\beta$ and the properties shown in (1). This yields a contradiction and, hence, Lemma 10 follows.

For the proof of Lemma 12 we will use the following lemma shown in [17], which is a straightforward consequence of Janson's inequality [10].

Lemma 14 The probability that the binomial random graph $\mathcal{G}\left(m, \frac{1}{8}\right)$ with $m \geq 253$ vertices and edge probability $1 / 8$ does not contain a copy of $K_{4}$ is bounded from above by $\exp \left(-2^{-11} m^{2}\right)$.

Proof of Lemma 12; We set

$$
\vartheta=2^{-17} \quad \text { and } \quad c=\frac{\vartheta}{4}
$$

and choose $\gamma>0$ such that

$$
3 h(2 \gamma)<\vartheta
$$

Let $\alpha, \beta$ and $c^{\prime \prime}$ be given by Lemma 10 We may also assume that

$$
3 \sqrt{h(6 \alpha)}+6 h(6 \alpha)<\vartheta / 2,
$$


as choosing $\alpha$ smaller we will only have to eventually increase $n_{0}$. Again, it is sufficient to keep in mind that

$$
0<\alpha, \beta \ll \gamma \ll \vartheta=2^{-17} .
$$

Due to Lemma 10 we have

$$
\left|\mathcal{F}_{n}^{\prime}(\alpha) \backslash \mathcal{F}_{n}^{\prime \prime}(\alpha, \beta)\right| \leq 2^{e\left(B_{n}\right)-c^{\prime \prime} n^{3}},
$$

and we estimate $\left|\mathcal{F}_{n}^{\prime \prime}(\alpha, \beta) \backslash \mathcal{F}_{n}^{\prime \prime \prime}(\alpha, \beta)\right|$ as follows.

Let $H \in \mathcal{F}_{n}^{\prime \prime}(\alpha, \beta) \backslash \mathcal{F}_{n}^{\prime \prime \prime}(\alpha, \beta)$ and $X_{H} \dot{\cup} Y_{H}$ be an arbitrary locally minimal $\alpha$-good partition. Consider a subset $S \in\left(\begin{array}{c}X_{H} \\ 3\end{array}\right) \dot{\cup}\left(\begin{array}{c}Y_{H} \\ 3\end{array}\right)$. Deleting $S$ from $V(H)$, we obtain a Fano-free hypergraph $H^{\prime}$ on $n-3$ vertices, where $V\left(H^{\prime}\right)=[n] \backslash S$. Note that for every $H \in \mathcal{F}_{n}^{\prime \prime}(\alpha, \beta) \backslash \mathcal{F}_{n}^{\prime \prime \prime}(\alpha, \beta)$ there exists a hypergraph $H^{\prime} \in \mathcal{F}_{n-3}$ such that $H$ can be obtained from $H^{\prime}$ in the following way. For $H^{\prime} \in \mathcal{F}_{n-3}$ we choose a set $S$ of 3 vertices, which we connect in an appropriate manner, so that the resulting hypergraph is in $\mathcal{F}_{n}^{\prime \prime}(\alpha, \beta) \backslash \mathcal{F}_{n}^{\prime \prime \prime}(\alpha, \beta)$.

We can choose the set $S$, the partition of $H^{\prime}$ and the set which contains $S$ in at most

$$
\left(\begin{array}{l}
n \\
3
\end{array}\right) 2^{n-3}
$$

ways. Note that the implicit partition of $H^{\prime}$ chosen that way is not necessarily locally minimal. Only after we add $S$ back, we obtain a locally minimal $\alpha$-good partition $\left(X_{H}, Y_{H}\right)$. Since $H \in \mathcal{F}_{n}^{\prime \prime}(\alpha, \beta)$ we infer from Lemma 10 that every vertex in $S$ has at most $\gamma n^{2}$ neighbors in its own partition class. This again bounds the number of ways for choosing these hyperedges by

$$
\left(\sum_{j=0}^{\gamma n^{2}-1}\left(\begin{array}{c}
n \\
2 \\
j
\end{array}\right)\right)^{3} \leq\left(\begin{array}{c}
n \\
2 \\
\gamma n^{2}
\end{array}\right)^{3} .
$$

For every vertex in $S$ we have at most $2^{n^{2} / 4}$ possibilities for choosing edges with one more end in the same partition as $S$ and the other end in the other partition class, this gives us at most

$$
2^{3 n^{2} / 4}
$$

ways to choose that type of hyperedges. The last estimate concerns the number of ways we can connect our triple $S$ to the other partition class, say $Y$, without creating any single copy of $K_{4}$, which is contained in the joint link of the vertices from $S$. Here we use Lemma 14. For every vertex $v$ in $S$ we can choose its link graph $L_{Y}(v)$ in at most $2^{\left(\begin{array}{c}|Y| \\ 2\end{array}\right)}$ ways. However, since the joint link of three vertices in $S$ contains no $K_{4}$, we infer from Lemma 14 , that there are at most

$$
2^{3\left(\begin{array}{c}
|Y| \\
2
\end{array}\right)} \exp \left(-2^{-11}|Y|^{2}\right)<2^{3\left(\begin{array}{c}
|Y| \\
2
\end{array}\right)-|Y|^{2} / 2^{11}}
$$

ways. 
Combining the above estimates and

$$
n / 4 \leq|Y| \leq n / 2+2 \sqrt{h(6 \alpha)} n,
$$

we obtain

$$
\begin{aligned}
& \left|\mathcal{F}_{n}^{\prime \prime}(\alpha, \beta) \backslash \mathcal{F}_{n}^{\prime \prime \prime}(\alpha, \beta)\right| \leq\left(\begin{array}{c}
n \\
3
\end{array}\right) 2^{n-3} \cdot\left(\begin{array}{c}
n \\
2 \\
\gamma n^{2}
\end{array}\right) 2^{3} 2^{3 n^{2} / 4} 2^{3\left(\begin{array}{c}
|Y| \\
2
\end{array}\right)-|Y|^{2} / 2^{11}}\left|\mathcal{F}_{n-3}\right| \\
& \text { 最 } 2^{3 \log n+n+3 h(2 \gamma) n^{2} / 2+9 n^{2} / 8+\vartheta n^{2} / 2-n^{2} / 2^{15}}\left|\mathcal{F}_{n-3}\right| \\
& \text { 罗 } 2^{\delta_{1}\left(B_{n-2}\right)+\delta_{1}\left(B_{n-1}\right)+\delta_{1}\left(B_{n}\right)+\vartheta n^{2}-n^{2} / 2^{16}}\left|\mathcal{F}_{n-3}\right| \\
& \text { 国 } 2^{\delta_{1}\left(B_{n-2}\right)+\delta_{1}\left(B_{n-1}\right)+\delta_{1}\left(B_{n}\right)-\vartheta n^{2}}\left|\mathcal{F}_{n-3}\right| \\
& \text { 是 } 2^{-\vartheta n^{2}} \cdot 2^{n-2} \cdot\left|\mathcal{B}_{n}\right| \text {. }
\end{aligned}
$$

Since

$$
\left|\mathcal{F}_{n}\right| \stackrel{\text { 1) }}{\leq} 2\left|\mathcal{B}_{n}\right| \leq 2^{e\left(B_{n}\right)+n+1},
$$

it follows from 9 , that

$$
\left|\mathcal{F}_{n}^{\prime}(\alpha) \backslash \mathcal{F}_{n}^{\prime \prime \prime}(\alpha, \beta)\right| \leq 2^{e\left(B_{n}\right)-c n^{2}}
$$

due to the choice of $c$.

\section{A construction}

Note that the error terms obtained in Lemmas 8 and 10 are of the order $2^{-\Omega\left(n^{3}\right)}$, while Lemma 12 only yields $2^{-\Omega\left(n^{2}\right)}$. The construction below indicates that Lemma 12 cannot be improved to obtain a similar bound. More precisely, we show that the number of the nonbipartite Fano-free hypergraphs is

$$
|\operatorname{Forb}(n, F)|-\left|\mathcal{B}_{n}\right|=2^{-\Theta\left(n^{2}\right)}\left|\mathcal{B}_{n}\right|
$$

Thus, for that many hypergraphs the algorithm $\operatorname{Color}(H)$ will perform the brute force search for an optimal coloring of the vertices. Indeed, (1) implies

$$
\frac{|\operatorname{Forb}(n, F)|-\left|\mathcal{B}_{n}\right|}{\left|\mathcal{B}_{n}\right|} \leq 2^{-\Omega\left(n^{2}\right)},
$$

while the construction below shows $|\operatorname{Forb}(n, F)|-\left|\mathcal{B}_{n}\right| \geq\left|\mathcal{B}_{n}\right| 2^{-O\left(n^{2}\right)}$.

The rough idea is to take a random balanced bipartite hypergraph on $n-9$ vertices, and then add some additional hyperedges incident to some of the remaining 9 vertices, such that almost surely the resulting hypergraph is not bipartite. A routine application of Chernoff's inequality gives then 10 .

Let $H^{\prime}$ be a random balanced bipartite 3-uniform hypergraph with classes $A, B$ such that $|A|+|B|=$ $n-9$. Let $v_{1}, \ldots, v_{9}$ be new vertices, let $G_{1}, \ldots, G_{6} \in \mathcal{G}(A, 1 / 2)$, i.e. $G_{i}$ 's are random graphs on the vertex set $A$. We define the hypergraph $H$ to have

$$
A \dot{\cup} B \dot{\cup}\left\{v_{1}, \ldots, v_{9}\right\}
$$


as a vertex set, and we define the hyperedge set of $H$ to be:

$$
\begin{aligned}
E(H):=E\left(H^{\prime}\right) \dot{\cup}\left\{e \cup\left\{v_{i}\right\}: e \in E\left(G_{i}\right), i \in\right. & {[6]\} \dot{\cup} } \\
& \left\{\left\{v_{1}, v_{2}, v_{7}\right\},\left\{v_{3}, v_{4}, v_{8}\right\},\left\{v_{5}, v_{6}, v_{9}\right\},\left\{v_{7}, v_{8}, v_{9}\right\}\right\} .
\end{aligned}
$$

Note that $H-v_{7} v_{8} v_{9}$ is bipartite, and moreover, since the hyperedges incident with $v_{7}, v_{8}$ and $v_{9}$ are disjoint, $H$ must be Fano-free. It is easy to show that with high probability, $H$ is not bipartite. In fact, w.h.p. a proper coloring of $H-v_{7} v_{8} v_{9}$ is unique (up to permutation of the two colors).

\section{Concluding Remarks}

We presented a deterministic algorithm that colors in $O\left(n^{5} \log ^{2} n\right)$ average running time every Fano-free hypergraph properly, which together with (1) implies also the class of bipartite 3-uniform hypergraphs.

Instead of asking for a deterministic algorithm, we could use results of Frieze and Kannan [6, 7], or a more recent one by Fischer, Matsliah and Shapira [5] to design randomized algorithms with better running time that color the same classes of hypergraphs properly with high probability. Note that the bottleneck in our algorithm was Step 2 of the algorithm Partition $(H, \alpha)$, which employed an application of the algorithmic version of the weak hypergraph regularity lemma due to Czygrinow and Rödl [2], that required $O\left(n^{5} \log ^{2} n\right)$ time. However, note that the while-loop of the same algorithm $\operatorname{Partition}(H, \alpha)$ has (naively implemented) a running time of $O\left(n^{5}\right)$.

It would be interesting to find faster randomized and deterministic algorithms for Corollary 2 Another interesting question, which might improve the running time is to find better deterministic algorithms for the weak hypergraph regularity lemma. In fact, in the case of graph regularity lemma an optimal algorithm was found in [13].

\section{Acknowledgements}

We would like to thank Asaf Shapira, Anusch Taraz and anonymous referees for useful suggestions. 


\section{References}

[1] H. Chen and A. Frieze, Coloring bipartite hypergraphs, Integer programming and combinatorial optimization (Vancouver, BC, 1996), Lecture Notes in Comput. Sci., vol. 1084, Springer, Berlin, 1996, pp. 345-358.

[2] A. Czygrinow and V. Rödl, An algorithmic regularity lemma for hypergraphs, SIAM J. Comput. 30 (2000), no. 4, 1041-1066 (electronic).

[3] I. Dinur, O. Regev, and C. Smyth, The hardness of 3-uniform hypergraph coloring, Combinatorica 25 (2005), no. 5, 519-535.

[4] M. E. Dyer and A. M. Frieze, The solution of some random NP-hard problems in polynomial expected time, J. Algorithms 10 (1989), no. 4, 451-489.

[5] E. Fischer, A. Matsliah, and A. Shapira, Approximate hypergraph partitioning and applications, 48th Annual IEEE Symposium on Foundations of Computer Science (FOCS 2007), October 20-23, 2007, Providence, RI, USA, Proceedings, IEEE Computer Society, 2007, pp. 579-589.

[6] A. Frieze and R. Kannan, The regularity lemma and approximation schemes for dense problems, Proceedings of the 37th Annual IEEE Symposium on Foundations of Computing, 1996, pp. 12-20.

[7] __ Quick approximation to matrices and applications, Combinatorica 19 (1999), no. 2, 175220.

[8] Z. Füredi and M. Simonovits, Triple systems not containing a Fano configuration, Combin. Probab. Comput. 14 (2005), no. 4, 467-484.

[9] V. Guruswami, J. Håstad, and M. Sudan, Hardness of approximate hypergraph coloring, SIAM J. Comput. 31 (2002), no. 6, 1663-1686 (electronic).

[10] S. Janson, Poisson approximation for large deviations, Random Structures Algorithms 1 (1990), no. 2, 221-229.

[11] P. Keevash and B. Sudakov, The Turán number of the Fano plane, Combinatorica 25 (2005), no. 5, 561-574.

[12] Y. Kohayakawa, B. Nagle, V. Rödl, and M. Schacht, Weak hypergraph regularity and linear hypergraphs, J. Combin. Theory Ser. B 100 (2010), no. 2, 151-160.

[13] Y. Kohayakawa, V. Rödl, and L. Thoma, An optimal algorithm for checking regularity., SIAM J. Comput. 32 (2003), no. 5, 1210-1235.

[14] Ph. G. Kolaitis, H. J. Prömel, and B. L. Rothschild, $K_{l+1}$ free graphs: asymptotic structure and a 0-1 law, Trans. Amer. Math. Soc. 303 (1987), no. 2, 637-671.

[15] M. Krivelevich, R. Nathaniel, and B. Sudakov, Approximating coloring and maximum independent sets in 3-uniform hypergraphs, J. Algorithms 41 (2001), no. 1, 99-113. 
[16] L. Lovász, Coverings and coloring of hypergraphs, Proceedings of the Fourth Southeastern Conference on Combinatorics, Graph Theory, and Computing (Florida Atlantic Univ., Boca Raton, Fla., 1973) (Winnipeg, Man.), Utilitas Math., 1973, pp. 3-12.

[17] Y. Person and M. Schacht, Almost all hypergraphs without Fano planes are bipartite, Proceedings of the Twentieth Annual ACM-SIAM Symposium on Discrete Algorithms (C. Mathieu, ed.), ACM, 2009, pp. 217-226.

[18] __ An expected polynomial time algorithm for coloring 2-colorable 3-graphs, Electronic Notes in Discrete Mathematics, vol. 34, Elsevier, 2009, pp. 465-469.

[19] H. J. Prömel and A. Steger, Coloring clique-free graphs in linear expected time, Random Structures Algorithms 3 (1992), no. 4, 375-402.

[20] E. Szemerédi, Regular partitions of graphs, Problèmes combinatoires et théorie des graphes (Colloq. Internat. CNRS, Univ. Orsay, Orsay, 1976), Colloq. Internat. CNRS, vol. 260, CNRS, Paris, 1978, pp. 399-401.

[21] J. S. Turner, Almost all k-colorable graphs are easy to color, J. Algorithms 9 (1988), no. 1, 63-82.

[22] H. S. Wilf, Backtrack: an O(1) expected time algorithm for the graph coloring problem, Inform. Process. Lett. 18 (1984), no. 3, 119-121. 